\title{
INTRODUCCIÓN A LA EFICIENCIA ENERGÉTICA A TRAVÉS DEL DISEÑO BIOCLIMÁTICO \\ ESTUDIO DE CASOS, VERIFICACIONES CUANTITATIVAS Y MANEJO DE NORMATIVAS.
}

\section{INTRODUCTION TO ENERGY EFFICIENCY THROUGH BIOCLIMATIC DESIGN CASE STUDIES, QUANTITATIVE VERIFICATIONS AND REGULATORY MANAGEMENT.}

\author{
Mgt. Arq. Panvini María José, Arq. Saldi Romina \\ Asignatura Optativa de un semestre asociada a la carrera de grado. \\ Facultad de Arquitectura, Planeamiento y Diseño. Universidad Nacional de Rosario, Argentina. \\ mjpanvini@yahoo.com.ar, rominasaldi@hotmail.com
}

\section{RESUMEN}

Desde el Espacio Curricular Optativo "Introducción a la eficiencia energética para viviendas" se busca internalizar en el alumno la necesidad de concebir la idea proyectual a través de un pensamiento integral que contemple las pautas de diseño con criterios de arquitectura pasiva. $Y$ vincular los temas teóricos impartidos en las diferentes materias de la carrera de Arquitectura en relación a la eficiencia energética para ser aplicados en la práctica proyectual de forma concreta.

El trabajo práctico debe cumplir con ciertos ítems que se resuelven parcial y metodológicamente a medida que se imparte la teoría. Se trabaja sobre tres tipologías de viviendas unifamiliares en PB implantadas en una zona bioclimática a elección. El alumno debe analizarlos según los principios y estrategias climáticas para llegar a determinar qué tipología se adapta mejor de acuerdo a los criterios y recomendaciones de diseño pasivo para dicha zona. Implica verificar los indicadores dimensionales, energéticos y la calidad térmica edilicia de la vivienda. Mediante la modalidad de seminario de integración que consta de una síntesis gráfica, cuantitativa se procede al debate e intercambio de ideas y reflexiones finales.

Los objetivos señalados desde la enseñanza-aprendizaje fueron logrados con éxito, siendo los temas teóricos aplicados correctamente. Se evidenció el uso de herramientas digitales, imágenes termográficas y modelados en 3D.

\section{ABSTRACT}

From the Optional Curriculum Subject "Introduction to energy efficiency for housing", the student seeks to internalize the need to conceive the project idea through an integral thinking that contemplates design guidelines with passive architecture criteria. And link theoretical subjects taught in the different subjects of Architecture career in relation to energy efficiency to be applied in project practice in a concrete way.

Practical work must comply with certain items that are partially and methodologically resolved as theory is taught. We work on three types of family homes on ground floor implanted in a bioclimatic zone of choice. The student must analyze them according to climate principles and strategies to determine which typology best suits according to the criteria and recommendations of passive design for that area. It implies verifying dimensional, energetic indicators and building thermal quality of house. Through the modality of integration seminar that consists of a graphic, quantitative synthesis, the debate and exchange of ideas and final reflections are carried out.

The objectives set from the teaching-learning were successfully achieved, with the theoretical issues being applied correctly. The use of digital tools, thermographic images and 3D modeling was evident. 
PALABRAS CLAVES: ARQUITECTURA PASIVA, CONFORT, ENERGÍA, NORMAS, RESILIENCIA.

KEY WORDS: PASSIVE ARCHITECTURE, COMFORT, ENERGY, NORMS, RESILIENCE.

\section{Artículo RECIBIDO: 21/07/19 | Artículo ACEPTADO: 11/11/19}

\section{INTRODUCCIÓN.}

"Si la arquitectura habrá de tener relevancia en el futuro, será necesario cambiar las reglas arquitectónicas establecidas durante las últimas décadas y tratar de entender cómo se puede proyectar con la naturaleza y no en contra de ella. Los edificios actuales, como los tradicionales, deben aprovechar las energías naturales del sol y del viento, incorporándolas a través de su diseño arquitectónico".

GERALD FOLEY ${ }^{17}$

Se puede decir que una vivienda es energéticamente eficiente cuando, a través de un diseño bioclimático pasivo, se reduce o minimiza el consumo de energía. Este hecho no implica renunciar al objetivo de lograr ambientes confortables.

Los edificios son importantes consumidores de energía y los modos en que son diseñados, construidos, utilizados, demolidos y sustituidos forman parte sustancial de las lógicas generales de organización urbana. Por estos motivos la asignatura optativa a la que pertenece el ejercicio práctico tiene por objetivos internalizar en el estudiante la toma de conciencia de la problemática bioambiental y la necesidad de concebir la idea proyectual a través de un pensamiento integral que contemple las pautas de diseño con criterios de arquitectura pasiva. Éstos tendrán relación con la eficiencia energética para ser luego aplicados en la práctica proyectual de forma concreta y fehacientemente. En este contexto, una edificación debe ser considerada como un "sistema" que, a través de estrategias proyectuales, logre el confort térmico sin recurrir al uso del acondicionamiento artificial.

La tipología elegida junto con las características termo físicas de los elementos constructivos de las envolventes y las propiedades de los materiales son aspectos que el alumno debe conocer y aplicar en sus propuestas de diseño pasivo para optimizar el comportamiento térmico en determinadas zonas bioclimáticas.

Si una vivienda está bien concebida, sus pérdidas de energía no serán excesivas, el consumo energético resultará comparativamente bajo y las emisiones contaminantes que producen gases de efecto invernadero también se reducirán, logrando de esta forma el objetivo de referencia.

\section{METODOLOGÍA.}

El Espacio Curricular Optativo está integrado por un equipo docente interdisciplinar y cuenta con la presencia de más de setenta alumnos. Se aborda un cursado presencial y se desarrolla un trabajo práctico integrador por grupos de alumnos de cuatro integrantes.

${ }^{17}$ Evans, J.M. y Schiller, S. (1988). Diseño Ambiental y Arquitectura Solar. Buenos Aires. Argentina: Ediciones Facultad de Arquitectura, Diseño y Urbanismo. Universidad de Buenos Aires. (pp. 5). 
El trabajo práctico es presentado por los docentes el primer día abordando progresivamente lo puntos que integran el avance a cumplir luego de la teoría impartida sobre cada uno de ellos (Fig. 4). Se incorpora también el uso de los EVA Entornos Virtuales de Aprendizaje mediante la plataforma moodle que dispone la facultad: http://moodle.fapyd.unr.edu.ar/course/view.php?id=164.

Allí se comparte la documentación de las clases teóricas y una serie de archivos de consulta, facilitado por el grupo de profesores y asesores externos.

La metodología del trabajo práctico consiste en el estudio de tres casos de diferentes tipologías de viviendas unifamiliares. Se trata de proyectos de planta baja del tipo compacto (Fig. 1), otro compacto con patio (Fig. 2) y uno de tipología abierta (Fig. 3).

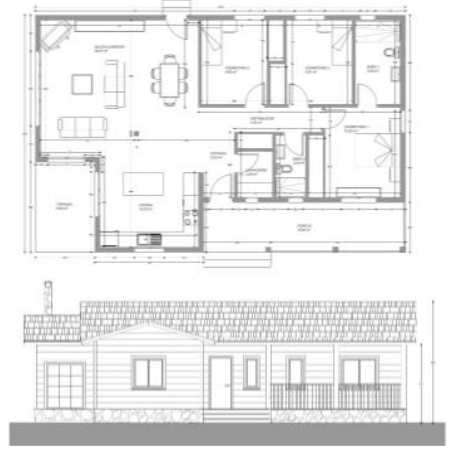

Figura 1. Tipología compacta

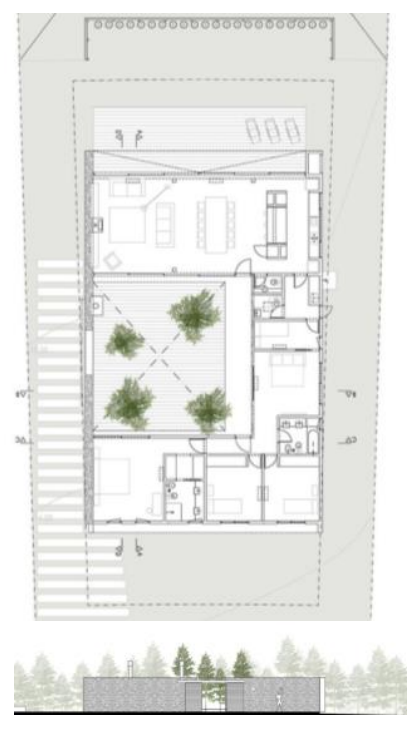

Figura 2. Tipología compacta con patio

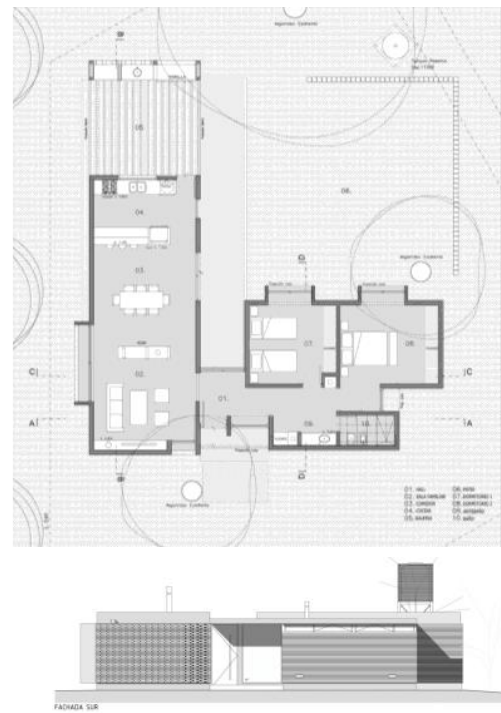

Figura 3. Tipología abierta

Al momento de iniciar el proceso de análisis de la vivienda es imprescindible conocer las condiciones ambientales del entorno en que ésta se emplazará, tomando en cuenta tanto los aspectos positivos como negativos que el clima ofrece.

El estudiante implanta en un terreno a elección ubicado en una determinada zona bioclimática y analiza las tres viviendas según los principios y estrategias de acuerdo a los criterios y recomendaciones de diseño pasivo para dicha zona. Determinar qué tipología se adapta mejor trae consigo verificar los indicadores dimensionales, energéticos y la calidad térmica edilicia, es decir, las pérdidas de energía en función del clima. Esto implica el cumplimiento de siete ítems que se resuelven parcial y metodológicamente a medida que se imparten los temas teóricos de la materia. Durante el cursado se van generando comprobaciones y avances parciales de las indagaciones (Fig. $5)$.

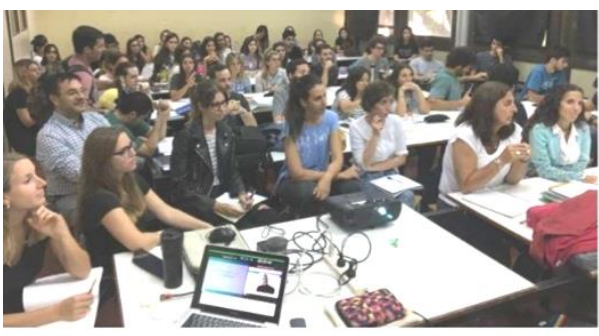

Figura 4. Clase inicial

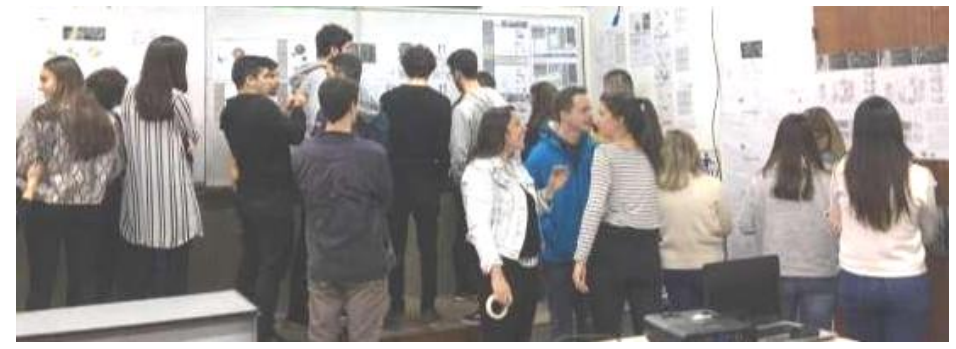

Figura 5. Encuentro avances parciales 
Luego de analizar y verificar las tres tipologías de viviendas mediante los nuevos conocimientos y procedimientos aprendidos y/o ya conocidos, justificación de por medio, el estudiante opta por la tipología que mejor se adecue a la zona bioclimática adoptada. La elección surge de las comprobaciones y conclusiones obtenidas, decidiendo por la tipología que menos intervenciones y/o modificaciones demanden para adecuarse a la región a implantarse, siempre teniendo en cuenta que las viviendas son proyectos pre diseñados. Esto implica no proyectarlos de cero sino que, desde un análisis crítico, sugerir posibles recomendaciones y/o estrategias de diseño pasivo que hagan posible su adecuación con el fin de obtener mejores resultados para conseguir UNA VIVIENDA ENERGÉTICAMENTE EFICIENTE.

Mediante la modalidad de seminario de integración, que consta de una exposición gráfica y oral, se procede al debate e intercambio de ideas y reflexiones finales. En base a los resultados obtenidos se exponen los trabajos para apreciación de la comunidad educativa en la galería de muestras de la Facultad de Arquitectura, Planeamiento y Diseño de la UNR. (Fig. 6).
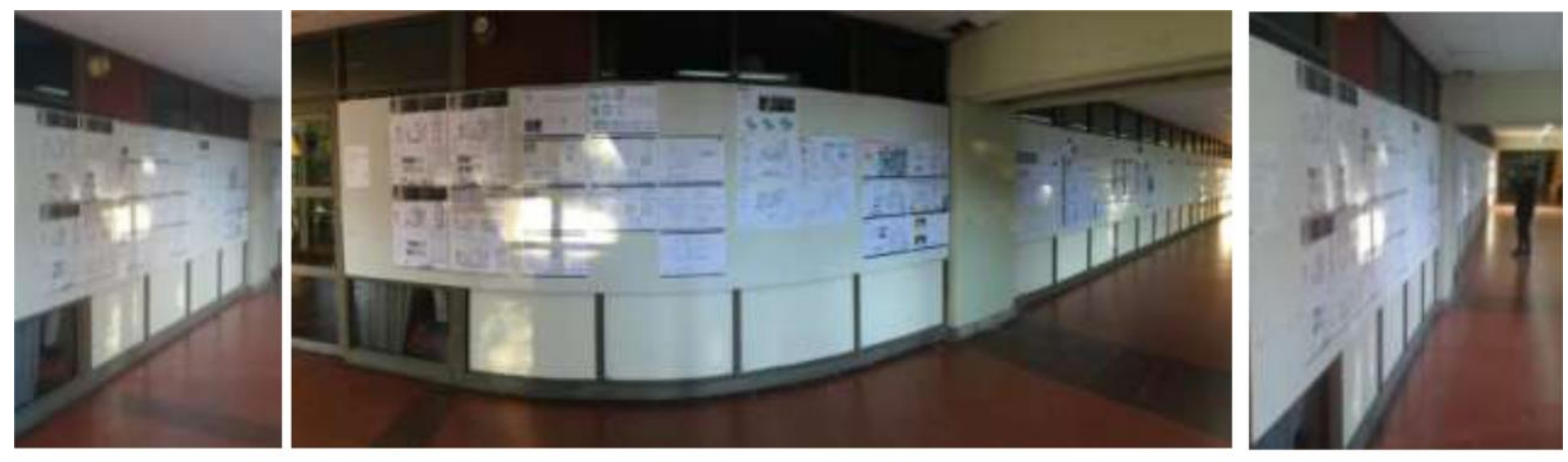

Figura 6. Galería de muestras de la Facultad de Arquitectura, Planeamiento y Diseño de la UNR

\section{DESARROLLO.}

El desarrollo del trabajo práctico integrador consta de siete ítems a cumplir que se ejemplifican a continuación:

I- Análisis de los FACTORES GEOGRÁFICOS (latitudes, temperaturas mínimas, humedades relativas, vientos predominantes, precipitaciones, grados día de calefacción) que determinan las características climáticas de las distintas zonas bioambientales (Fig. 7)

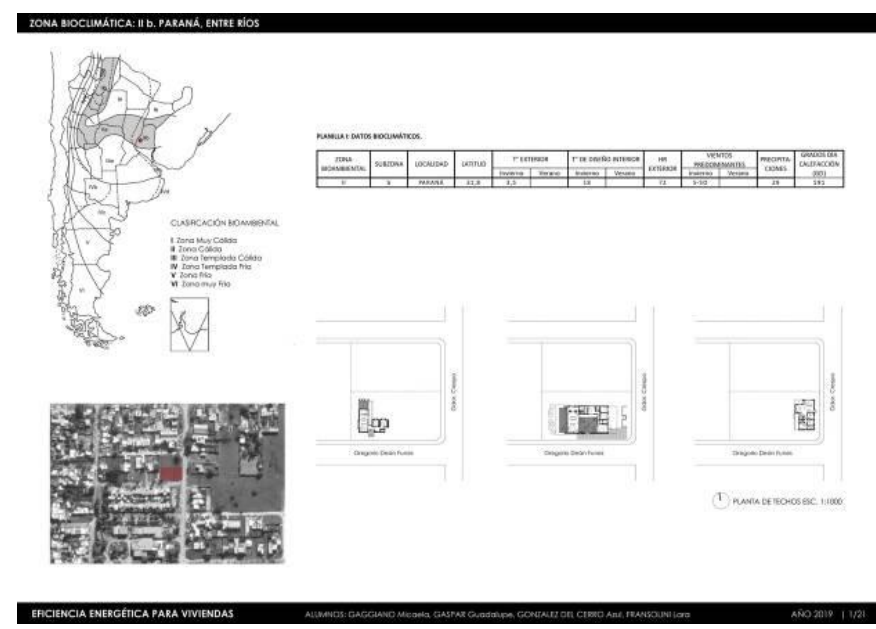

Figura 7. Autores Gaggiano, Gaspar, González del Cerro, Fransolini 
II- Estudio de las PAUTAS Y PRINCIPIOS GENERALES PARA EL DISEÑO BIOCLIMÁTICO: (Fig. 8)

- Implantación de la obra en el sitio,

- Ventilación natural,

- Orientaciones favorables,

- Asoleamiento mínimo,

- Disposición de los espacios interiores,

- Tamaño y orientación de las aberturas,

- Identificación de las protecciones solares,

- lluminación natural.
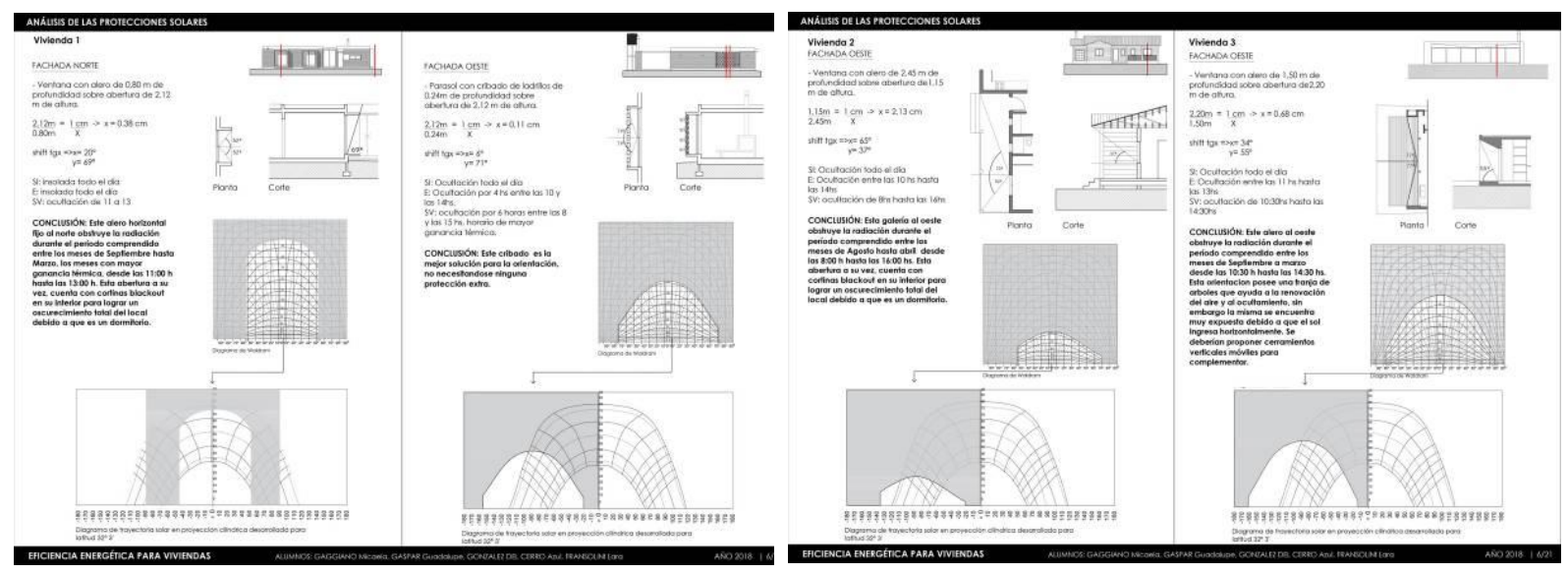

Figura 8. Autores Gaggiano, Gaspar, González del Cerro, Fransolini

III- Analizar los puntos anteriores según tablas confeccionadas por la cátedra: (Figs. 9 y 10).

- Recomendaciones de estrategias de diseño pasivo,

- Efectos del clima de las diferentes regiones en la forma de la vivienda,

- Criterios y recomendaciones de diseño bioclimático para el ordenamiento del conjunto,

- Criterios y recomendaciones de diseño bioclimático para el ordenamiento de la vivienda.

- Identificar y/o determinar las ESTRATEGIAS DE DISEÑO PASIVO adoptadas en cada tipología.
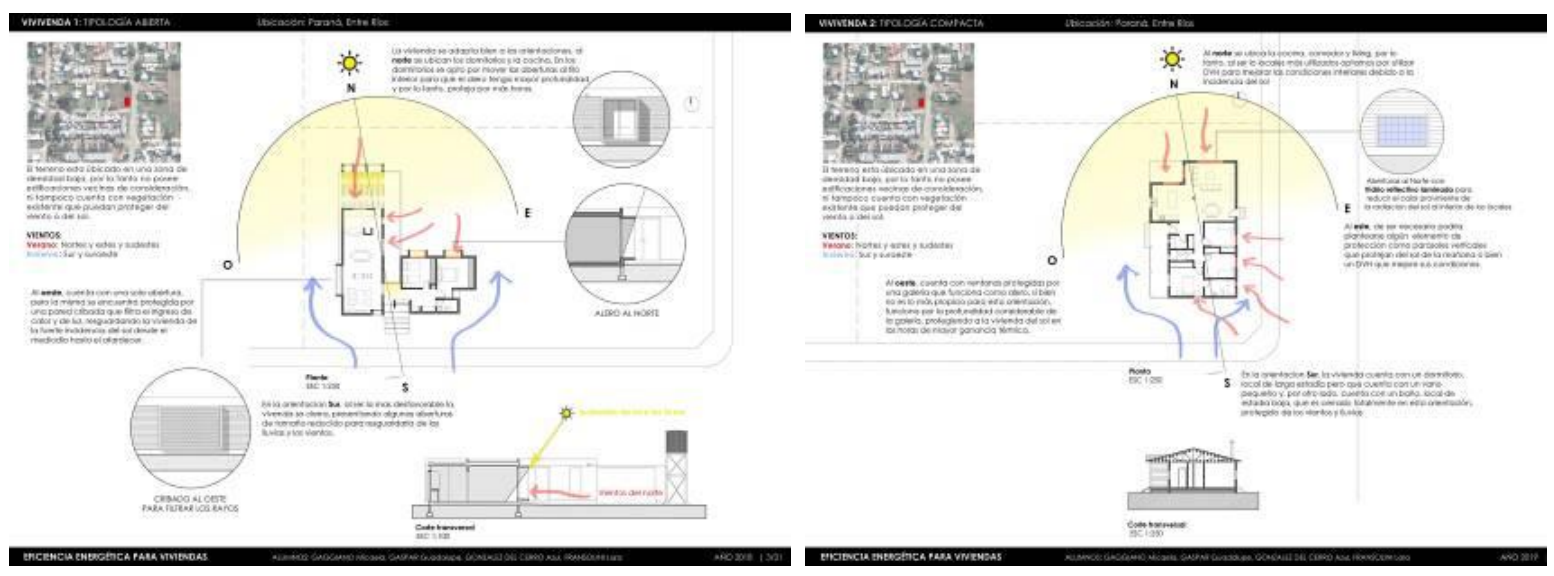

Figura 9. Autores Gaggiano, Gaspar, González del Cerro, Fransolini 


\section{INTRODUCCIÓN A LA EFICIENCIA ENERGÉTICA A TRAVÉS DEL DISEÑO BIOCLIMÁTICO}

Mgt. Arq. Panvini María José, Arq. Saldi Romina

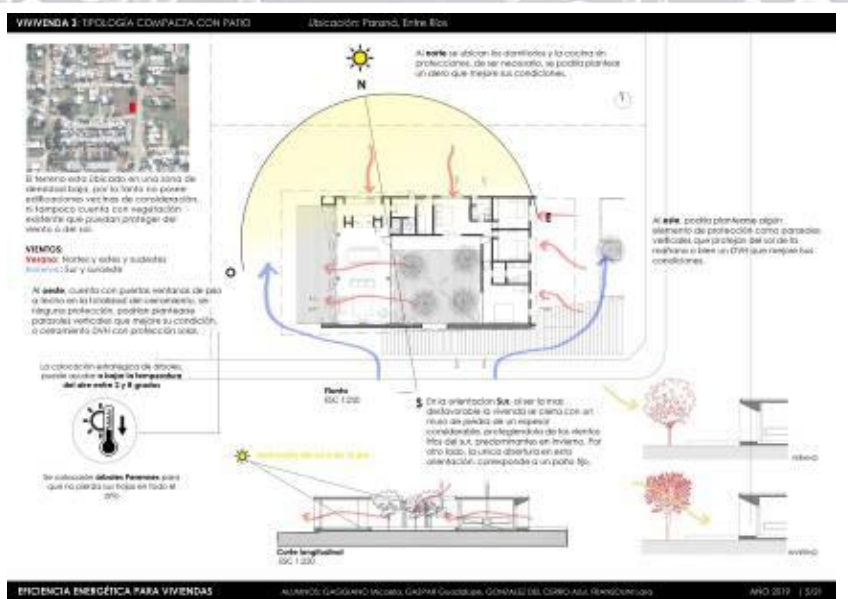

Figura 10. Autores Gaggiano, Gaspar, González del Cerro, Fransolini

IV- Evaluación de la FORMA ARQUITECTÓNICA en relación a la conservación de la energía: indicadores dimensionales (FAEP Factor Área Envolvente Piso: relación entre la superficie de la envolvente con respecto a la superficie de piso) (Fig. 11).

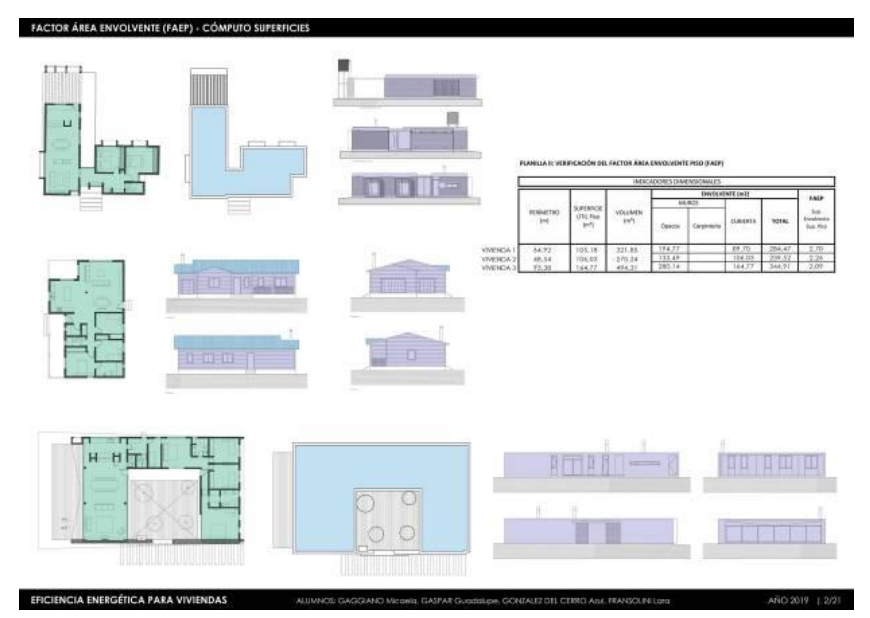

Figura 11. Autores Gaggiano, Gaspar, González del Cerro, Fransolini

V- Proponer soluciones de envolventes verticales y horizontales para las tres tipologías. Verificación del COMPORTAMIENTO ENERGÉTICO (transmitancia térmica de cerramientos horizontales y verticales opacos y vidriados, condensación superficial e intersticial y FES Factor de Exposición Solar) (Fig. 12).
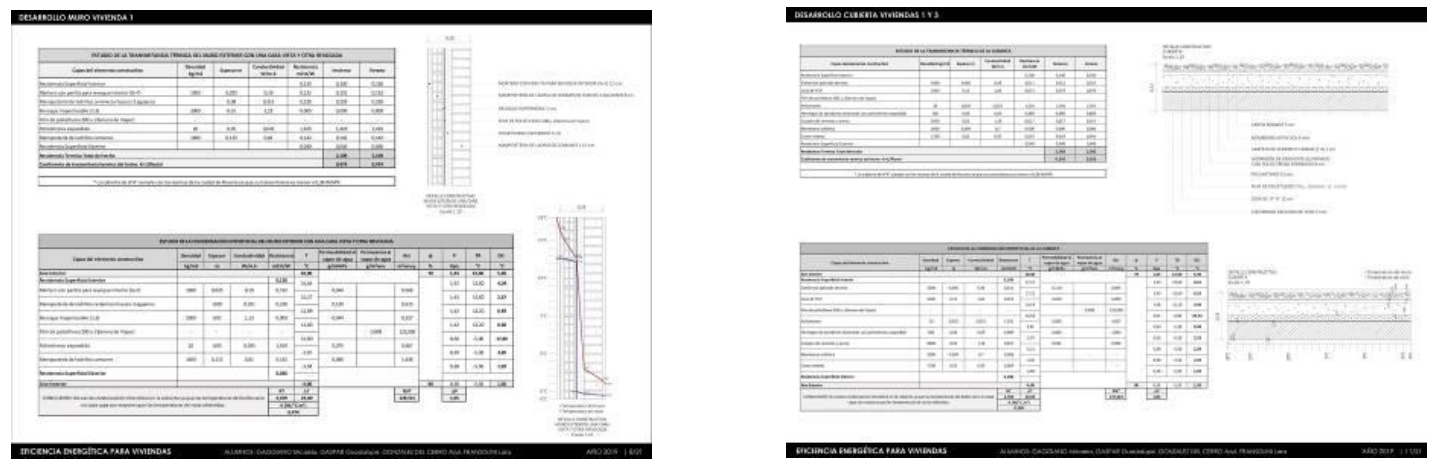

Figura 12. Autores Gaggiano, Gaspar, González del Cerro, Fransolini 
VI- Cálculo de la PÉRDIDA VOLUMÉTRICA GLOBAL DE CALOR (coeficiente G).

VII- Determinación de la CARGA TÉRMICA ANUAL (Kwh/año).

Para llevar a cabo las cuantificaciones correspondientes, el alumno las determina y verifica según Normas IRAM 11601 (1996), 11604 (2001), 11605 (1996), 11625 (2000), 11630 (2000) y/o Ordenanza Municipal Rosario 8757 (2011) según la zona bioambiental. Rosario, pertenece a la zona bioambiental III "a". Fue la primera ciudad de Argentina en implementar medidas para la disminución de energía del acondicionamiento térmico tanto en calefacción como en refrigeración en la construcción.

Los valores no debieran exceder los máximos admisibles fijados en las normas para dar cumplimiento con el ahorro energético. Más allá de los resultados obtenidos, dichos valores le proveen al estudiante un indicador numérico de la EFICACIA TÉRMICA de cada proyecto, transformándose en un auxiliar de interés en el proceso del diseño constructivo.

La determinación de la carga térmica anual (Kwh/año), como último paso, permite conocer cuánta energía primaria se necesita anualmente para mantener la vivienda de manera constante a $18^{\circ}, 20^{\circ} \mathrm{o}$ $22^{\circ} \mathrm{C}$ (según grados días de calefacción adoptado), y así poder comparar las cargas térmicas anuales de las viviendas calefaccionadas y conocer el costo de energía necesario para mantener ese confort, posibilitando estimar el ahorro energético derivado de las posibles mejoras sobre las características térmicas que las mismas implicaran.

\section{CONCLUSIONES Y REFLEXIONES}

Concluimos como equipo docente del espacio curricular optativo que el objetivo apuntado desde la enseñanza - aprendizaje fue logrado con éxito por los estudiantes. Los mismos se mostraron entusiastas en el uso de herramientas digitales como ser incorporación de imágenes termográficas y modelados en 3D (Figura 13).

L os temas teóricos impartidos fueron aplicados de forma correcta. Gran parte de los grupos recurrió a la utilización de los recursos naturales como estrategias de diseño pasivo (Ejemplo: uso de la vegetación para disminuir el efecto de las brisas predominantes). 


\section{INTRODUCCIÓN A LA EFICIENCIA ENERGÉTICA A TRAVÉS DEL DISEÑO BIOCLIMÁTICO}

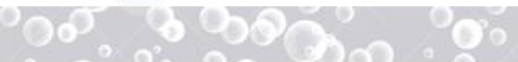
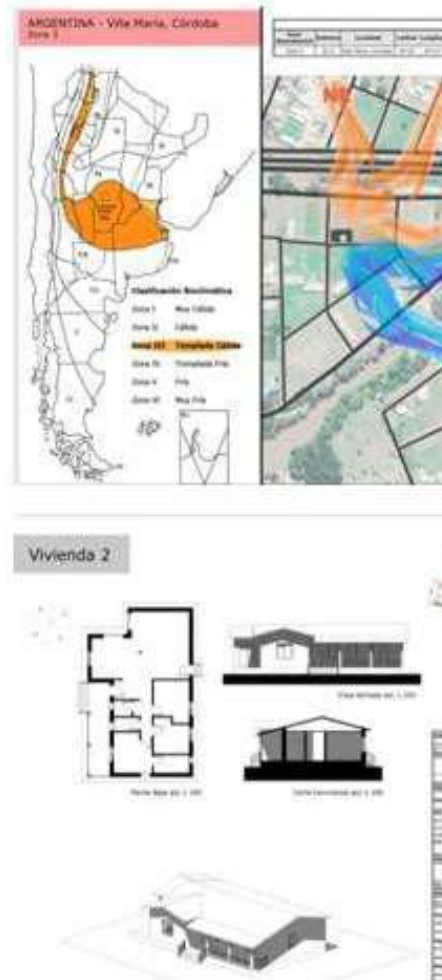
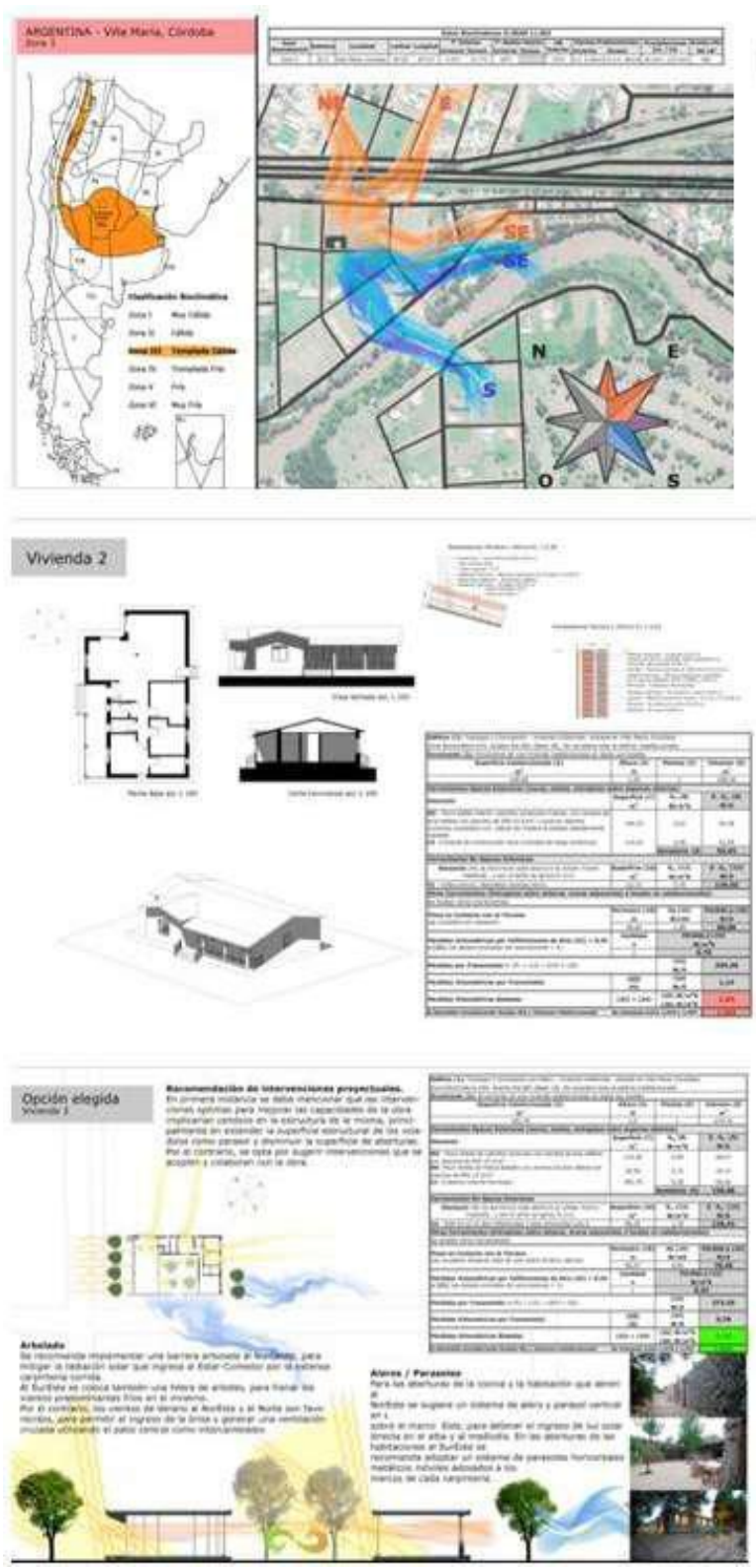

Mgt. Arq. Panvini María José, Arq. Saldi Romina

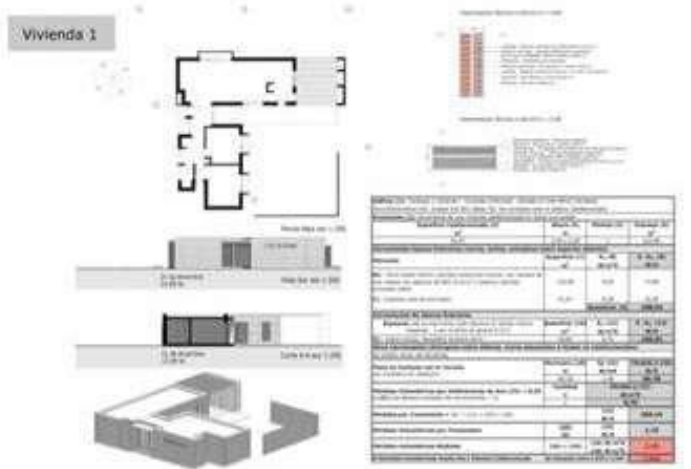

Vivienda 3
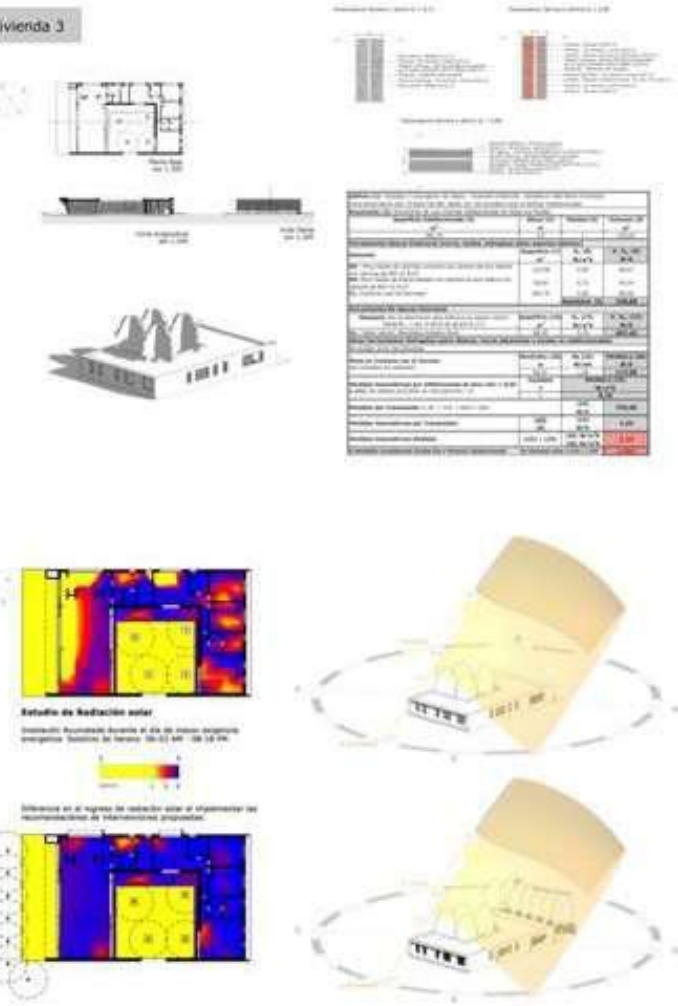

Figura 13. Autores Arino, Busti.Feito, Corrado, Micheli

\section{BIBLIOGRAFÍA}

IRAM (1996). Norma IRAM 11601. Acondicionamiento térmico de edificios. Método de cálculo. Propiedades térmicas de los componentes y elementos de construcción en régimen estacionario. Buenos Aires. Argentina.

IRAM (2001). Norma IRAM 11604. Aislamiento térmico de edificios. Verificación de sus condiciones higrotérmicas. Ahorro de energía en calefacción. Coeficiente volumétrico $\mathrm{G}$ de pérdidas de calor. Cálculo y valores límites. Buenos Aires. Argentina. 
IRAM (1996). Norma IRAM 11605. Acondicionamiento térmico de edificios. Condiciones de habitabilidad en viviendas. Valores máximos de transmitancia térmica en cerramientos opacos. Buenos Aires. Argentina.

IRAM (2000). Norma IRAM 11625. Verificación del riesgo de condensación de vapor de agua. Buenos Aires. Argentina.

IRAM (2000). Norma IRAM 11630. Aislamiento térmico de edificios. Verificación de sus condiciones higrotérmicas. Verificación del riesgo de condensación de vapor de agua superficial e intersticial en puntos singulares de muros exteriores, pisos y techos de edificios en general. Buenos Aires. Argentina.

Bielsa, M. E.; Cortés, A.; Greppi, O.; López, N. (2011). Ordenanza 8757. Aspectos higrotérmicos y demanda energética de las construcciones. Rosario. Argentina.

Fein, M. (2013). Decreto 985. Aplicación reglamentaria de aspectos higrotérmicos y eficiencia energética

de construcciones. Rosario. Argentina.

Panvini, M. J.; Saldi, R. (2019). Tabla 1 de elaboración propia: Recomendaciones de estrategias de diseño

pasivo. Rosario. Argentina.

Panvini, M. J.; Saldi, R. (2019). Tabla 2 de elaboración propia: Efectos del clima de las diferentes regiones

en la forma de la vivienda. Rosario. Argentina.

Panvini, M. J.; Saldi, R. (2019). Tabla 3 de elaboración propia: Criterios y recomendaciones de diseño bioclimático para el ordenamiento del conjunto. Rosario. Argentina.

Panvini, M. J.; Saldi, R. (2019). Tabla 4 de elaboración propia: Criterios y recomendaciones de diseño bioclimático para el ordenamiento de la vivienda. Rosario. Argentina.

Municipalidad de Rosario (2013). Planilla Valores de FES Factor de Exposición de Solar. Rosario. Argentina. 